\title{
Comparison between Dual-Energy X-ray Absorptiometry and Bioelectrical Impedance Analyses for Accuracy in Measuring Whole Body Muscle Mass and Appendicular Skeletal Muscle Mass
}

\author{
Seo Young Lee ${ }^{1,2}$, Soyeon Ahn ${ }^{3}{ }^{10}$, Young Ji Kim ${ }^{1}$, Myoung Jin Ji ${ }^{1}$, Kyoung Min Kim ${ }^{1}$, \\ Sung Hee Choi ${ }^{1}$, Hak Chul Jang ${ }^{1}$ (D) and Soo Lim ${ }^{1, *}$ \\ 1 Department of Internal Medicine, Seoul National University College of Medicine and Seoul National \\ University Bundang Hospital, Seongnam 13620, Korea; danaehyuk@naver.com (S.Y.L.); \\ vernade@hanmail.net (Y.J.K.); hahajmj@hanmail.net (M.J.J.); kyoungmin02@gmail.com (K.M.K.); \\ shchoimd@gmail.com (S.H.C.); janghak@snu.ac.kr (H.C.J.) \\ 2 Department of Internal Medicine, Mediplex Sejong Hospital, Incheon 21080, Korea \\ 3 Division of Statistics, Medical Research Collaborating Center, Seoul National University Bundang Hospital, \\ Seongnam 13620, Korea; ahnsoyeon@gmail.com \\ * Correspondence: limsoo@snu.ac.kr; Tel.: +82-31-787-7035
}

Received: 10 April 2018; Accepted: 31 May 2018; Published: 7 June 2018

\begin{abstract}
We evaluate the accuracy of whole body muscle mass (WBMM) and appendicular skeletal muscle mass (ASMM) assessed by bioelectrical impedance analysis (BIA) using an InBody770 machine (InBody, Seoul, Korea) referenced to dual-energy X-ray absorptiometry (DXA) in 507 people (mean age $63.7 \pm 10.8$ years, body mass index (BMI) $25.2 \pm 3.5 \mathrm{~kg} / \mathrm{m}^{2}$ ). Mean WBMMs measured by BIA and DXA were $49.3 \pm 6.6 \mathrm{~kg}$ and $46.8 \pm 6.5 \mathrm{~kg}$ in men and $36.1 \pm 4.7 \mathrm{~kg}$ and $34.0 \pm 4.8 \mathrm{~kg}$ in women, respectively. The respective effect sizes and $95 \%$ confidence intervals (CIs) for the difference were 2.49 (2.22-2.76) for men, and 2.12 (1.91-2.33) for women. Mean ASMMs measured by BIA and DXA were $22.1 \pm 3.3 \mathrm{~kg}$ and $19.9 \pm 3.2 \mathrm{~kg}$ in men, and $15.3 \pm 2.5 \mathrm{~kg}$ and $13.5 \pm 2.2 \mathrm{~kg}$ in women, respectively. The respective effect sizes and 95\% CIs for the difference were 2.26 (2.10-2.41) for men and 1.75 (1.65-1.87) for women. The BIA clearly overestimated WBMM by $2.28 \mathrm{~kg}$ and ASMM by $1.97 \mathrm{~kg}$ compared with DXA. Using BMI, gender, and fat percentage, we derive equations that improved the residuals to $<2 \mathrm{~kg}$ between methods from $38.29 \%$ to $85.91 \%$ for WBMM and $52.78 \%$ to 97.02\% for ASMM.
\end{abstract}

Keywords: bioelectrical impedance analysis; dual-energy X-ray absorptiometry; muscle mass

\section{Introduction}

Most developed and developing countries have been facing rapid increases in the elderly population. A decrease in muscle mass or sarcopenia is an important contributor to declining health in this group [1-4]. In this situation, it is important to measure muscle mass accurately for the management of sarcopenia, which causes many metabolic disorders, physiological problems, and functional impairments that eventually lead to disability in elderly populations [5-8]. Therefore, preservation or buildup of muscle mass is becoming an important health issue for this age group, and accurate measurement of muscle mass is the first step for adequate prevention and treatment of sarcopenia.

The common imaging modalities to measure amount of muscle mass are computed tomography (CT) and magnetic resonance imaging (MRI). Both methods are useful to measure muscle 
cross-sectional area precisely $[9,10]$. However, CT is expensive and carries the risk of radiation exposure particularly for whole-body assessment. Although there is such no risk of radiation exposure in MRI, its high cost, long examination time, noise, regional variations in accessibility, and limited use with metal implants prevent its use as a routine screening method for elderly persons. By contrast, dual-energy X-ray absorptiometry (DXA) has several advantages, such as its easy applicability, low cost, and low radiation exposure [11]. Therefore, DXA is regarded as the gold standard method for analyzing body composition at the molecular level. In this method, the body is divided into three components: fat mass, lean mass, and bone mineral content [12-14]. DXA has been used for measuring muscle mass in several studies [15-17]. Body composition in a specific compartment can also be measured with recent upgraded software for DXA. However, there is still a small amount of radiation exposure. In addition, extremely obese people are not suitable for this method because they exceed weight limitations.

Several other methods for body composition assessment, such as bioelectrical impedance analysis (BIA) and sonography, have now been developed. Among them, BIA has drawn attention because it is easily applicable, imposes no burden on subjects, involves easy to move and relatively cheap equipment, and most importantly, poses no radiation hazard [18,19]. Using BIA, information on body composition can be obtained simply with the subject in a standing position in a short time (less than $3 \mathrm{~min}$ ). Thus, BIA is widely used not only in specialized facilities, but also in fitness and health checkup centers [20].

So far, several studies have been conducted to investigate the usefulness of BIA in measuring body composition, but mainly in people of European origin [16,17]. Moreover, another study with Mexican subjects had a small number of participants and used old machines that lacked accuracy [21]. However, there are not many studies using BIA with Asians. Body composition differs between Caucasians and Asians: Asians have a greater tendency for obesity at a lower BMI than Caucasians [22]. This difference is linked to differences in adipokines such as adiponectin and leptin, which are associated with adiposity and muscle mass [23,24]. Therefore, ethnicity-specific equations are required for precise estimation of muscle mass by BIA.

BIA devices have improved from single-frequency to multi-frequency and multi-segmental instruments using more electrodes, which has helped improve the accuracy [25]. Recently, more advanced technology has been used to evaluate both muscle mass and fat mass [26,27]. In this study, we investigated the accuracy of whole body muscle mass (WBMM) and appendicular skeletal muscle mass (ASMM) measures obtained with a BIA machine in subjects in the standing position for DXA reference values in Korean adults with a wide age range. In addition, we aimed to derive equations for better estimating muscle mass using relevant variables.

\section{Methods}

\subsection{Subjects}

We consecutively included 504 Koreans who had undergone both DXA and BIA over a short-term interval. Study participants were selected from the outpatient clinic of Seoul National University Bundang Hospital (SNUBH), Korea, from May 2015 to July 2017. We included physically active subjects aged 20-90 years. Subjects were excluded if they had severe debilitating diseases such as liver cirrhosis; stoke with paralysis; cerebral palsy; malignancy; a medical history of major operations, including organ removal and amputation; or were on hemodialysis or peritoneal dialysis. Written informed consent was received from all subjects. This study was approved by the Institutional Review Board of SNUBH (IRB no. B-1704-390-007) and complied with the principles of the Declaration of Helsinki and its contemporary amendments.

\subsection{Measurement of Clinical and Biochemical Parameters}

Anthropometric parameters were measured in all study participants. Height (in $\mathrm{cm}$ ) was measured to the nearest $0.1 \mathrm{~cm}$ and weight (in $\mathrm{kg}$ ) was measured to the nearest $0.1 \mathrm{~kg}$ with the 
subject wearing light clothing. Body mass index (BMI) was calculated as weight (in kg) divided by height (in $\mathrm{m}$ ) squared. Waist circumference was measured with the subject in a standing position at the umbilical point at the end of expiration by trained examiners. Blood pressure was measured using an automated blood pressure machine. Clinical data, including age, gender, comorbidity (including diabetes mellitus (DM), and hypertension), smoking habits, and alcohol consumption, were collected from each participant. Medication usage, such as diuretics and thiazolidinedione (TZD), was also investigated because these drugs can affect fluid status in the body.

Blood samples were collected in the morning after $12 \mathrm{~h}$ fasting to measure biochemical variables such as fasting plasma glucose, blood urea nitrogen (BUN), creatinine, total protein, albumin, white blood cell, hemoglobin, hematocrit, platelet counts, lipid profiles, and liver enzymes.

\subsection{Muscle Mass Measurement Using DXA}

For DXA, a Hologic Horizon W machine (Hologic Inc., Bedford, MA, USA) was used to measure WBMM and ASMM in the study participants. Before the DXA scan, participants were asked to remove all metal objects and to change into a gown. Scanning was performed with the subject supine, and the scan time was within $15 \mathrm{~min}$. ASMM was calculated as the sum of the muscle mass in both arms and legs.

\subsection{Muscle Mass Estimation by BIA}

For BIA, an Inbody770 machine (Inbody Co., Seoul, Korea) was used to estimate muscle mass $[28,29]$. The measurement was performed with the subject in a standing position grasping the electrodes with both hands abducted from the mid-body. There was a total of eight electrodes: two for each foot and two for each hand. The measurement comprised two combinations: $z$-axis at frequencies of $1,5,50,250$, and $500 \mathrm{kHz}$ for impedance and $x$-axis at frequencies of 5,50 , and $250 \mathrm{kHz}$ for reactance. Impedance was measured for five segments of the body: trunk, right and left arms, and right and left legs. Resistance was measured at four surface tactile electrodes placed on the dorsal surface of the hand and foot by the BIA generator; the formula $\mathrm{V}=\varrho x$ height ${ }^{2} / \mathrm{resistance}$ was used to derive muscle mass mathematically [30]. Resistance is the resistance that occurs when alternating current passes through the body water, and reactance indicates the resistance of the cell membrane through which the alternating current passes. Impedance is the vector sum of these two components. In the InBody770 device, the reactance was calculated using a trigonometric formula with these parameters. Using the manufacturer's algorithm, fat and muscle masses of the total body, arms, and legs were calculated separately. WBMM is the sum of the muscle masses in the whole body and ASMM is the sum of the lean mass of the arms and legs. The time gap between BIA measures and DXA scans was $\sim 0.5$ days.

The InBody720, which had the same hardware as the InBody770, demonstrated a strong correlation with DXA (iDXA GE, Bedford, MA, USA) in ASMM (Pearson correlation coefficients 0.944 and 0.903 , and standard error of estimate $1.051 \mathrm{~kg}$ and $0.927 \mathrm{~kg}$ in men and women, respectively) [26]. A previous study showed a strong intra-class correlation $(r=0.9995)$ for the estimation of body fat percent using an InBody720 device [27]. Based on these data, InBody devices received a domestic medical device manufacturing item certificate by the Ministry of Food and Drug Safety of Korea.

Because BIA is sensitive to hydration status, participants were asked to refrain from alcohol consumption or vigorous exercise for $24 \mathrm{~h}$ before the measurement. Both BIA and DXA were measured in the morning after overnight fasting to make the hydration status as uniform as possible.

To minimize the contact noise, we cleaned the contacting surface of the electrodes with an alcohol swab before every measurement. In addition, the current electrode and voltage electrode were separated from each other by a total of eight electrodes because of the structure of the hand. Starting the measurement at the wrist and ankle, where the flow of current and measurement of voltage meet, minimized the influence of the finger and palm, which have high contact resistance. Using these 
methods, we were able to maintain high reproducibility in the body composition measurements using InBody devices [27].

\subsection{Statistical Analysis}

Data are presented as the mean \pm standard deviation (SD) or number $(n)$ with percentage. Pearson's correlation analysis was used to test concordance between BIA and DXA muscle measurements. Agreement in muscle mass measurements between BIA and DXA was checked using the Bland-Altman method, but this was not used for cross-validation. To analyze differences among the subgroups according to age, gender, BMI, body fat, and other clinical features, we used the Student's $t$-test and analysis of variance (ANOVA). We considered several possible models based on clinical importance and feasibility. Continuous variables were fitted with linear term or polynomial terms. Possible interaction terms were also included. A linear model was built with clinically relevant and statistically significant predictors, and linear or quadratic terms were considered during the model specification process. Normality assumptions were visually assessed through quantile-quantile plot, and multicollinearity of predictors was checked using a variance inflation factor. We derived the final model that had small Bayesian information criterion (BIC) value and not too many variables by parsimonious rule. For the final models, variance inflation factors (VIFs) were calculated to rule out collinearity. We used IBM SPSS Statistics v. 22.0 for Windows (IBM Corp., Armonk, NY, USA) and R statistical software v. 3.1.1 for Windows (Foundation for Statistical Computing, Vienna, Austria), and values of $p<0.05$ were considered significant.

\section{Results}

\subsection{Baseline Clinical Characteristics of the Study Population}

The baseline characteristics of the subjects $(n=507)$ are shown in Table 1 . The mean age of men $(n=213)$ and women $(n=294)$ was almost the same. For muscle mass assessment, men had a greater WBMM than women by about $13.0 \mathrm{~kg}$ either using the DXA (46.8 $\pm 6.5 \mathrm{~kg}$ vs. $34.0 \pm 4.8 \mathrm{~kg})$ or BIA methods $(49.3 \pm 6.6 \mathrm{~kg}$ vs. $36.1 \pm 4.7 \mathrm{~kg})$. The BIA approach estimated muscle mass to be greater than did DXA: $2.3 \mathrm{~kg}$ greater for WBMM and $2.0 \mathrm{~kg}$ greater for ASMM. Whole body fat mass and fat percentage assessed by BIA was greater in women than in men.

Table 1. Anthropometric and biochemical characteristics and comorbidity of the study populations $(n=507)$.

\begin{tabular}{cccc}
\hline & Men $(\boldsymbol{n = 2 1 3})$ & Women $(\boldsymbol{n = 2 9 4 )}$ & ${ }^{*} \boldsymbol{p}$ \\
\hline Age (years) & $64.1 \pm 1.3$ & $63.4 \pm 10.3$ & 0.511 \\
Height $(\mathrm{cm})$ & $168.6 \pm 5.8$ & $155.4 \pm 5.6$ & $<0.001$ \\
Weight $(\mathrm{kg})$ & $71.8 \pm 11.0$ & $60.9 \pm 10.2$ & $<0.001$ \\
BMI $\left(\mathrm{kg} / \mathrm{m}^{2}\right)$ & $25.2 \pm 3.1$ & $25.2 \pm 3.8$ & 0.919 \\
Waist circumference $(\mathrm{cm})$ & $88.9 \pm 6.3$ & $84.9 \pm 8.9$ & $<0.001$ \\
SBP $(\mathrm{mmHg})$ & $128.4 \pm 13.7$ & $127.2 \pm 13.9$ & 0.328 \\
DBP $(\mathrm{mmHg})$ & $74.7 \pm 10.1$ & $75.2 \pm 9.1$ & 0.565 \\
\hline Laboratory findings & & & \\
FPG $(70-110 \mathrm{mg} / \mathrm{dL})$ & $135.0 \pm 41.5$ & $117.6 \pm 33.9$ & $<0.001$ \\
HbA1c $(4.0-6.4 \%)$ & $7.1 \pm 1.4$ & $6.6 \pm 1.2$ & $<0.001$ \\
WBC $\left(4-10 \times 10^{3} / \mu \mathrm{L}\right)$ & $6.4 \pm 1.7$ & $5.6 \pm 1.6$ & $<0.001$ \\
Hemoglobin $(13-17 \mathrm{~g} / \mathrm{dL})$ & $14.6 \pm 1.5$ & $13.2 \pm 1.0$ & $<0.001$ \\
Hematocrit $(39-52 \%)$ & $43.1 \pm 4.1$ & $39.7 \pm 3.0$ & $<0.001$ \\
Platelet $\left(130-400 \times 10^{3} / \mu \mathrm{L}\right)$ & $211.5 \pm 52.9$ & $243.4 \pm 53.9$ & $<0.001$ \\
Total cholesterol $(0-240 \mathrm{mg} / \mathrm{dL})$ & $161.1 \pm 36.1$ & $180.8 \pm 40.2$ & $<0.001$ \\
Triglycerides $(0-200 \mathrm{mg} / \mathrm{dL})$ & $139.2 \pm 90.5$ & $132.4 \pm 65.6$ & 0.325 \\
HDL-cholesterol $(35-55 \mathrm{mg} / \mathrm{dL})$ & $47.5 \pm 10.5$ & $54.9 \pm 11.9$ & $<0.001$ \\
LDL-cholesterol $(0-130 \mathrm{mg} / \mathrm{dL})$ & $91.6 \pm 27.3$ & $102.1 \pm 29.9$ & $<0.001$ \\
\hline
\end{tabular}


Table 1. Cont.

\begin{tabular}{cccc}
\hline & Men $(\boldsymbol{n}=\mathbf{2 1 3})$ & Women $(\boldsymbol{n}=\mathbf{2 9 4})$ & ${ }^{*} \boldsymbol{p}$ \\
\hline BUN $(10-26 \mathrm{mg} / \mathrm{dL})$ & $17.4 \pm 11.4$ & $15.0 \pm 4.3$ & 0.001 \\
Creatinine $(0.70-1.40 \mathrm{mg} / \mathrm{dL})$ & $0.9 \pm 0.2$ & $0.7 \pm 0.1$ & $<0.001$ \\
eGFR (mL/min/1.73 m $\left.{ }^{2}\right)$ & $85.3 \pm 18.8$ & $90.5 \pm 19.3$ & 0.003 \\
Total protein $(6.0-8.0 \mathrm{~g} / \mathrm{dL})$ & $7.2 \pm 0.4$ & $7.3 \pm 0.4$ & 0.034 \\
Albumin (3.3-5.2 g/dL) & $4.4 \pm 0.3$ & $4.4 \pm 0.2$ & 0.053 \\
AST (1-40 IU/L) & $25.7 \pm 8.3$ & $27.2 \pm 15.0$ & 0.161 \\
ALT (1-40 IU/L) & $27.1 \pm 13.8$ & $26.1 \pm 20.1$ & 0.566 \\
\hline Muscle mass by DXA & & & \\
Whole body lean mass (kg) & $46.8 \pm 6.5$ & $34.0 \pm 4.8$ & $<0.001$ \\
Appendicular skeletal muscle mass (kg) & $19.9 \pm 3.2$ & $13.5 \pm 2.2$ & $<0.001$ \\
\hline Muscle mass by BIA & & & $<0.001$ \\
Whole body muscle mass (kg) & $49.3 \pm 6.6$ & $36.1 \pm 4.7$ & $<0.001$ \\
Appendicular skeletal muscle mass (kg) & $22.1 \pm 3.3$ & $15.3 \pm 2.5$ & $<0.001$ \\
\hline Fat mass by BIA & & & $<0.001$ \\
Fat mass (kg) & $19.6 \pm 5.7$ & $22.5 \pm 6.8$ &
\end{tabular}

Data are expressed as the mean \pm SD. BMI, body mass index; WC, waist circumference; SBP, systolic blood pressure; DBP, diastolic blood pressure; WBC, white blood cell; FPG, fasting plasma glucose; LDL, low-density lipoprotein; HDL, high-density lipoprotein; BUN, blood urea nitrogen; eGFR, estimated glomerular filtration rate; AST, aspartate transaminase; ALT, alanine transaminase; DXA, dual-energy x-ray absorptiometry; BIA, bioelectrical impedance analysis. ${ }^{*} p$ values by Student's $t$-test between men and women.

\subsection{Comparison of Muscle Mass Estimated by BIA with That Measured by DXA (Table 2)}

The WBMM and ASMM values estimated by BIA were highly correlated with those measured by DXA in the entire study group (both $r>0.97, p<0.01$ ). We then investigated whether differences between muscle masses measured by DXA and BIA were associated with anthropometric and biochemical parameters. Using ANOVA, the differences in WBMM between the two measurements were affected by gender, BMI, age, and body fat. Men had a larger difference in muscle mass assessments between the two methods than did women $(p<0.001)$. No gender differences were found in either the model using the interaction term gender or in the subgroup analysis stratified by gender (data not shown). Among the BMI categories, subjects with a lower BMI showed larger differences in WBMM assessments between the two methods, compared with those with a higher BMI (Figure 1). For age, the differences showed a U-shaped pattern: larger differences were found in people who were younger than 40 years and older than 60 years compared with those who were in their 40s and 50s.

For ASMM, the difference in its measurement between the two methods was found to be affected by gender: men showed larger differences than women $(2.3 \pm 1.1 \mathrm{~kg}$ vs. $1.8 \pm 0.9 \mathrm{~kg}, p<0.05)$ (Table 2$)$. Like WBMM, subjects with a lower BMI showed larger differences in ASMM assessments between the two methods, compared with those with a higher BMI, but it had marginal significance (Table 2 and Figure 1). In contrast to WBMM, age group and body fat did not affect the differences in ASMM measurements between the two methods. 
(a)

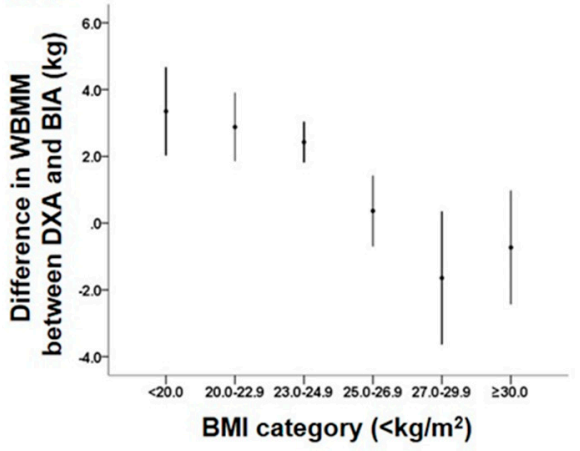

(c)

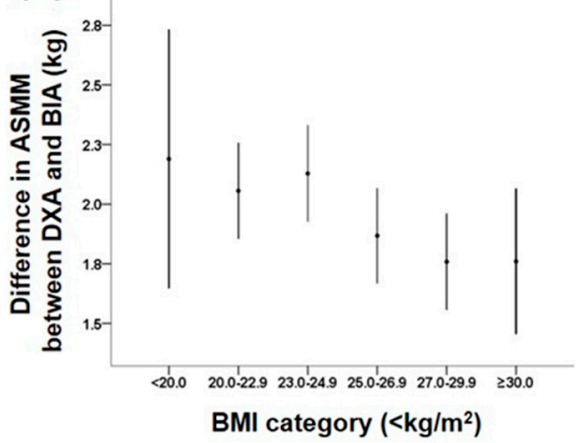

(b)

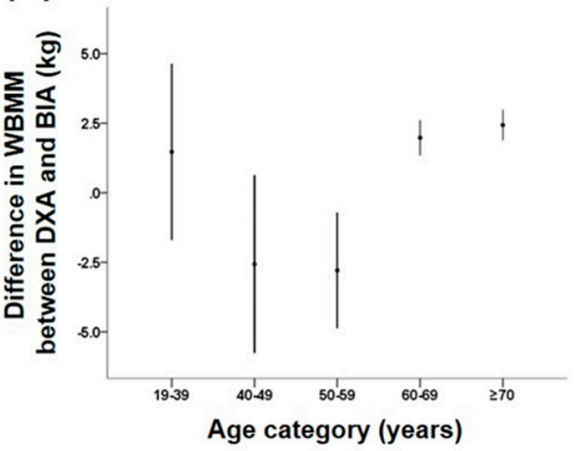

(d)

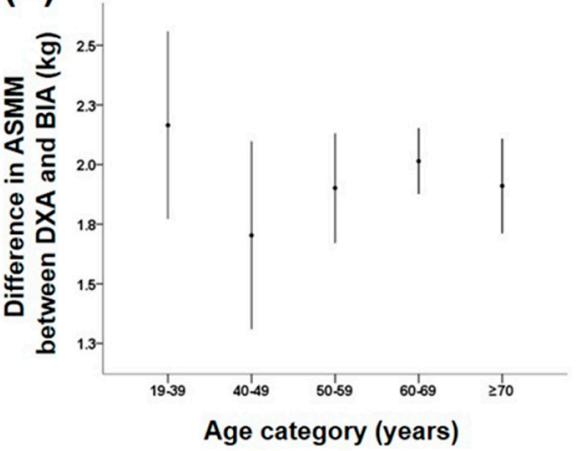

Figure 1. Differences in whole body lean muscle mass (WBMM) and appendicular skeletal muscle mass (ASMM) between DXA scans and BIA methods according to body mass index (BMI) and age categories. (a) WBMM by BMI group; (b) WBMM by age group; (c) ASMM by BMI group; and (d) ASMM by age group.

Table 2. Mean differences in muscle mass observed by dual-energy X-ray absorptiometry (DXA) and bioelectrical impedance analysis (BIA) according to gender, BMI, and age groups and body fat categories.

\begin{tabular}{|c|c|c|c|c|c|c|c|}
\hline & $\mathbf{N}$ & Mass by DXA (kg) & Mass by BIA (kg) & Difference (kg) & $* p$ & ${ }^{\dagger} p$ & ICC \\
\hline \multicolumn{8}{|c|}{ (a) Whole body muscle mass (WBMM) } \\
\hline Total & 507 & $39.4 \pm 8.4$ & $41.6 \pm 8.6$ & $2.3 \pm 2.0$ & $<0.001$ & & 0.972 \\
\hline Gender & & & & & & $<0.001$ & \\
\hline Men & 213 & $46.8 \pm 6.5$ & $49.3 \pm 6.6$ & $2.5 \pm 2.1$ & $<0.001$ & & 0.947 \\
\hline Women & 294 & $34.0 \pm 4.8$ & $36.1 \pm 4.7$ & $2.1 \pm 1.9$ & $<0.001$ & & 0.918 \\
\hline BMI $\left(\mathrm{kg} / \mathrm{m}^{2}\right)$ & & & & & & $<0.001$ & \\
\hline$<20$ & 22 & $30.4 \pm 4.7$ & $33.8 \pm 5.8$ & $3.4 \pm 2.1$ & $<0.001$ & & 0.936 \\
\hline $20-22.9$ & 112 & $35.3 \pm 6.7$ & $38.5 \pm 7.3$ & $3.2 \pm 1.7$ & $<0.001$ & & 0.975 \\
\hline $23-24.9$ & 97 & $38.2 \pm 6.9$ & $41.3 \pm 7.1$ & $3.0 \pm 1.6$ & $<0.001$ & & 0.973 \\
\hline $25-26.9$ & 137 & $39.8 \pm 7.0$ & $41.7 \pm 7.6$ & $1.9 \pm 2.0$ & $<0.001$ & & 0.965 \\
\hline $27-29.9$ & 75 & $43.1 \pm 9.0$ & $44.6 \pm 9.8$ & $1.5 \pm 1.8$ & $<0.001$ & & 0.986 \\
\hline$\geq 30$ & 47 & $47.0 \pm 10.2$ & $47.5 \pm 10.8$ & $0.4 \pm 2.1$ & $<0.001$ & & 0.982 \\
\hline Age (years) & & & & & & $<0.001$ & \\
\hline $19-39$ & 21 & $44.7 \pm 12.5$ & $47.7 \pm 12.9$ & $2.9 \pm 1.9$ & $<0.001$ & & 0.989 \\
\hline $40-49$ & 33 & $47.7 \pm 9.5$ & $49.3 \pm 9.7$ & $1.6 \pm 1.7$ & $<0.001$ & & 0.984 \\
\hline 50-59 & 69 & $42.9 \pm 8.7$ & $44.5 \pm 9.1$ & $1.6 \pm 2.5$ & $<0.001$ & & 0.963 \\
\hline $60-69$ & 228 & $37.7 \pm 7.5$ & $40.3 \pm 7.7$ & $2.6 \pm 1.9$ & $<0.001$ & & 0.963 \\
\hline$\geq 70$ & 142 & $37.2 \pm 6.8$ & $39.5 \pm 7.1$ & $2.2 \pm 2.0$ & $<0.001$ & & 0.961 \\
\hline Body fat $(\%) \ddagger$ & & & & & & 0.003 & \\
\hline Non-obese & 74 & $37.5 \pm 8.0$ & $40.5 \pm 8.4$ & $2.9 \pm 2.0$ & $<0.001$ & & 0.972 \\
\hline Obese & 420 & $39.6 \pm 8.5$ & $41.7 \pm 8.7$ & $2.2 \pm 2.0$ & $<0.001$ & & 0.972 \\
\hline
\end{tabular}


Table 2. Cont.

\begin{tabular}{|c|c|c|c|c|c|c|c|}
\hline & $\mathbf{N}$ & Mass by DXA (kg) & Mass by BIA (kg) & Difference (kg) & ${ }^{*} p$ & ${ }^{+} p$ & ICC \\
\hline \multicolumn{8}{|c|}{ (b) Appendicular skeletal muscle mass (ASMM) } \\
\hline Total & 507 & $16.2 \pm 4.1$ & $18.2 \pm 4.4$ & $2.0 \pm 1.1$ & $<0.001$ & \multirow{4}{*}{$<0.001$} & 0.972 \\
\hline Gender & & & & & & & \\
\hline Men & 213 & $19.9 \pm 3.2$ & $22.1 \pm 3.3$ & $2.3 \pm 1.1$ & $<0.001$ & & 0.939 \\
\hline Women & 294 & $13.5 \pm 2.2$ & $15.3 \pm 2.5$ & $1.8 \pm 0.9$ & $<0.001$ & & 0.928 \\
\hline BMI $\left(\mathrm{kg} / \mathrm{m}^{2}\right)$ & & & & & & \multirow[t]{7}{*}{0.093} & \\
\hline$<20$ & 22 & $12.2 \pm 2.4$ & $14.4 \pm 3.1$ & $2.2 \pm 1.3$ & $<0.001$ & & 0.932 \\
\hline $20-22.9$ & 112 & $14.4 \pm 3.3$ & $16.5 \pm 3.9$ & $2.1 \pm 1.1$ & $<0.001$ & & 0.973 \\
\hline $23-24.9$ & 97 & $15.8 \pm 3.3$ & $17.9 \pm 3.7$ & $2.1 \pm 1.0$ & $<0.001$ & & 0.968 \\
\hline $25-26.9$ & 137 & $16.3 \pm 3.6$ & $18.2 \pm 3.9$ & $1.9 \pm 1.1$ & $<0.001$ & & 0.958 \\
\hline $27-29.9$ & 75 & $17.8 \pm 4.7$ & $19.6 \pm 5.0$ & $1.8 \pm 0.9$ & $<0.001$ & & 0.985 \\
\hline$\geq 30$ & 47 & $19.5 \pm 5.3$ & $21.2 \pm 5.4$ & $1.8 \pm 1.0$ & $<0.001$ & & 0.981 \\
\hline Age (years) & & & & & & \multirow[t]{6}{*}{0.503} & \\
\hline $19-39$ & 21 & $19.2 \pm 6.5$ & $21.3 \pm 6.7$ & $2.2 \pm 0.9$ & $<0.001$ & & 0.991 \\
\hline $40-49$ & 33 & $20.4 \pm 4.7$ & $22.1 \pm 4.7$ & $1.7 \pm 1.1$ & $<0.001$ & & 0.971 \\
\hline 50-59 & 69 & $17.8 \pm 4.2$ & $19.7 \pm 4.5$ & $1.9 \pm 1.0$ & $<0.001$ & & 0.979 \\
\hline $60-69$ & 228 & $15.4 \pm 3.6$ & $17.5 \pm 4.0$ & $2.0 \pm 1.0$ & $<0.001$ & & 0.968 \\
\hline$\geq 70$ & 142 & $15.0 \pm 3.3$ & $17.0 \pm 3.9$ & $2.0 \pm 1.2$ & $<0.001$ & & 0.959 \\
\hline Body fat $(\%) \ddagger$ & & & & & & \multirow[t]{3}{*}{0.675} & \\
\hline Non-obese & 74 & $15.4 \pm 3.8$ & $17.4 \pm 4.5$ & $2.0 \pm 1.1$ & $<0.001$ & & 0.975 \\
\hline Obese & 420 & $16.3 \pm 4.2$ & $18.2 \pm 4.5$ & $2.0 \pm 1.1$ & $<0.001$ & & 0.972 \\
\hline
\end{tabular}

Data are expressed as the mean \pm SD. ${ }^{*} p$ values between DXA and BIA. ${ }^{\dagger} p$ values by one-way ANOVA for changes across groups. ${ }^{\ddagger}$ For men, non-obese $(<25.7 \%)$, obese $(\geq 25.7 \%)$; for women, non-obese $(<35.9 \%)$, obese $(\geq 36.0 \%)$.

The Bland-Altman plot for comparison between the two methods in the assessment of muscle mass showed a significant trend, which indicates increasing error with increase in muscle mass (Figure 2). We further investigated differences in WBMM and ASMM between the two methods according to subgroups classified as above or below a BMI of $25 \mathrm{~kg} / \mathrm{m}^{2}$ and an age of 50 years in men and women to compare their differences according to these categories (Supplementary Table S1). In both men and women, individuals with BMI $<25 \mathrm{~kg} / \mathrm{m}^{2}$ had larger differences in both WBMM and ASMM estimates than did those with BMI $\geq 25 \mathrm{~kg} / \mathrm{m}^{2}$ (all $p<0.01$ except for $p=0.089$ in women for WBMM). For the two age categories with a cutoff of 50 years, there were no significant trends in differences in WBMM and ASMM between the two methods.
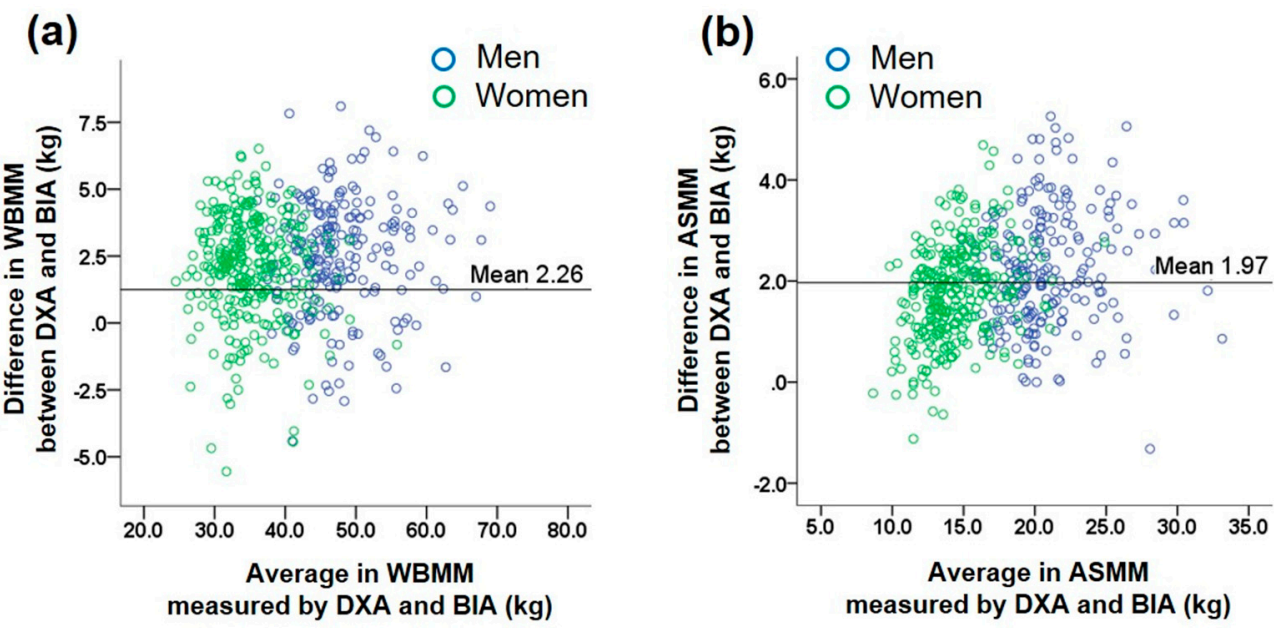

Figure 2. Bland-Altman plot for comparison between the two methods. (a) Whole body lean muscle mass (WBMM), (b) appendicular skeletal muscle mass (ASMM) in subjects who underwent DXA and BIA. Dotted horizontal lines are $95 \%$ confidence intervals. 
3.3. Subgroup Analysis of Mean Differences in Muscle Mass Estimates between DXA and BIA Methods According to Clinical Features (Table 3)

There were strong correlations for both WBMM and ASMM between the DXA and BIA methods (all $r>0.9, p<0.001$ ). For both WBMM and ASMM, hemoglobin levels, estimated glomerular filtration rate (eGFR), the presence of DM, and the use of diuretics or TZD did not affect the difference between the two methods.

Table 3. Pearson correlation coefficients between DXA and BIA muscle mass estimates in subgroups according to clinical features.

\begin{tabular}{|c|c|c|c|c|c|c|}
\hline \multicolumn{7}{|c|}{ (a) Whole Body Muscle Mass (WBMM) } \\
\hline & & $n$ & $B I A-D X A$ & $r$ & ${ }^{*} p$ & ${ }^{t} p$ \\
\hline \multirow{2}{*}{ Anemia } & $\mathrm{Hb} \geq 12 \mathrm{~g} / \mathrm{dL}$ & 457 & $2.33 \pm 1.93$ & 0.971 & $<0.001$ & \multirow[b]{2}{*}{0.734} \\
\hline & $\mathrm{Hb}<12 \mathrm{~g} / \mathrm{dL}$ & 50 & $1.93 \pm 2.26$ & 0.938 & $<0.001$ & \\
\hline \multirow{2}{*}{ Kidney function } & $\mathrm{eGFR} \geq 60$ & 480 & $2.25 \pm 1.91$ & 0.975 & $<0.001$ & \multirow{2}{*}{0.242} \\
\hline & $\begin{array}{c}\mathrm{eGFR}<60 \\
\mathrm{~mL} / \mathrm{min} / 1.73 \mathrm{~m}^{2}\end{array}$ & 27 & $2.80 \pm 2.18$ & 0.958 & $<0.001$ & \\
\hline \multirow{2}{*}{$\mathrm{DM}$} & $\mathrm{DM}(+)$ & 327 & $2.16 \pm 2.04$ & 0.971 & $<0.001$ & \multirow{2}{*}{0.157} \\
\hline & $\mathrm{DM}(-)$ & 180 & $2.48 \pm 1.70$ & 0.978 & $<0.001$ & \\
\hline \multirow{2}{*}{ Medication (1) } & Diuretics $(-)$ & 457 & $2.32 \pm 1.92$ & 0.975 & $<0.001$ & \multirow{2}{*}{0.187} \\
\hline & Diuretics $(+)$ & 50 & $1.83 \pm 1.96$ & 0.966 & $<0.001$ & \\
\hline \multirow{2}{*}{ Medication (2) } & $\mathrm{TZD}(-)$ & 477 & $2.28 \pm 1.92$ & 0.973 & $<0.001$ & \multirow{2}{*}{0.213} \\
\hline & TZD (+) & 30 & $2.06 \pm 1.72$ & 0.989 & $<0.001$ & \\
\hline \multicolumn{7}{|c|}{ (b) Appendicular Skeletal Muscle Mass (ASMM) } \\
\hline \multirow{2}{*}{ Anemia } & $\mathrm{Hb} \geq 12 \mathrm{~g} / \mathrm{dL}$ & 457 & $2.01 \pm 1.12$ & 0.967 & $<0.001$ & \multirow{2}{*}{0.617} \\
\hline & $\mathrm{Hb}<12 \mathrm{~g} / \mathrm{dL}$ & 50 & $1.92 \pm 1.12$ & 0.956 & $<0.001$ & \\
\hline \multirow{2}{*}{ Kidney function } & $\begin{array}{c}\mathrm{eGFR} \geq 60 \\
\mathrm{~mL} / \mathrm{min} / 1.73 \mathrm{~m}^{2}\end{array}$ & 480 & $2.01 \pm 1.11$ & 0.973 & $<0.001$ & \multirow{2}{*}{0.407} \\
\hline & $\begin{array}{c}\mathrm{eGFR}<60 \\
\mathrm{~mL} / \mathrm{min} / 1.73 \mathrm{~m}^{2}\end{array}$ & 27 & $2.32 \pm 1.13$ & 0.962 & $<0.001$ & \\
\hline \multirow{2}{*}{$\mathrm{DM}$} & $\mathrm{DM}(+)$ & 327 & $2.16 \pm 2.04$ & 0.971 & $<0.001$ & \multirow{2}{*}{0.697} \\
\hline & $\mathrm{DM}(-)$ & 180 & $2.48 \pm 1.70$ & 0.973 & $<0.001$ & \\
\hline \multirow{2}{*}{ Medication (1) } & Diuretics (-) & 457 & $2.03 \pm 1.12$ & 0.973 & $<0.001$ & \multirow{2}{*}{0.704} \\
\hline & Diuretics (+) & 50 & $1.92 \pm 1.12$ & 0.971 & $<0.001$ & \\
\hline \multirow{2}{*}{ Medication (2) } & TZD (-) & 477 & $2.01 \pm 1.13$ & 0.971 & $<0.001$ & \multirow{2}{*}{0.299} \\
\hline & $\mathrm{TZD}(+)$ & 30 & $1.82 \pm 1.14$ & 0.973 & $<0.001$ & \\
\hline
\end{tabular}

${ }^{*} p$ values by Pearson correlation analysis between DXA and BIA. ${ }^{\dagger} p$ values by Fisher's $z$-test between correlation coefficients. BIA, bioelectrical impedance analysis; DXA, dual-energy X-ray absorptiometry; Hb, hemoglobin; eGFR, estimated glomerular filtration rate; DM, diabetes mellitus; $\mathrm{HbA1c}$, glycated hemoglobin; TZD, thiazolidinedione.

\subsection{Prediction of DXA Estimation of Muscle Mass Measured by BIA Method by Multivariate Regression Models (Table 4)}

To best predict the DXA estimation of muscle mass by BIA measurement, we fitted multiple models and chose the model that satisfied minimal penalty functions while maintaining small variables and VIF $<10$. Finally, we generated equations using significant covariates including gender, BMI, and fat percent obtained by BIA. The final models were derived as follows:

$\mathrm{WBMM}=4.01+0.28 \times \mathrm{BMI}+(-2.93) \times$ Gender $+0.61 \times$ WBMM-by-BIA $+0.001 \times$ $(\mathrm{WBMM}-\mathrm{by}-\mathrm{BIA})^{2}+0.10 \times$ fat percent-by-BIA;

$\mathrm{ASMM}=5.07+0.26 \times \mathrm{BMI}+(-1.19) \times$ Gender $+0.24 \times$ ASMM-by-BIA $+0.01 \times$ $(\mathrm{ASMM}-\mathrm{by}-\mathrm{BIA})^{2}+(-0.06) \times$ fat percent-by-BIA. 
Table 4. Multivariate regression models for the prediction of DXA muscle mass measured by BIA.

\begin{tabular}{cccccccc}
\hline \multicolumn{3}{c}{ Whole Body Muscle Mass } & \multicolumn{3}{c}{ Appendicular Skeletal Muscle Mass } \\
\hline & Co-Efficient & \multicolumn{2}{c}{$\mathbf{9 5 \%}$ CI } & & Co-Efficient & 95\% CI \\
\hline & \multicolumn{3}{c}{ Lower } & Upper & & Lower & Upper \\
\hline Intercept & 4.01 & 0.80 & 7.22 & Intercept & 5.07 & 3.67 & 6.47 \\
BIA-WBMM & 0.61 & 0.48 & 0.74 & BIA-ASMM & 0.24 & 0.12 & 0.37 \\
(BIA-WBMM) ${ }^{2}$ & 0.00 & 0.00 & 0.00 & (BIA-ASMM) & 0.01 & 0.01 & 0.01 \\
BMI & 0.28 & 0.19 & 0.38 & BMI & 0.26 & 0.21 & 0.31 \\
Gender & -2.93 & -3.37 & -2.48 & Gender & -1.19 & -1.45 & -0.93 \\
BIA-fat percent & 0.10 & 0.05 & 0.15 & BIA-fat percent & -0.06 & -0.08 & -0.04 \\
\hline
\end{tabular}

WBMM, whole body muscle mass; ASMM, appendicular skeletal muscle mass; BIA, bioelectrical impedance analysis; DXA, dual-energy X-ray absorptiometry; CI, confidence interval.

Before and after applying the models, the differences between the two methods were compared based on the percentage of the value where the residual was less than $2 \mathrm{~kg}$. After applying the model, for ASMM; the percentage of residuals $<2 \mathrm{~kg}$ increased from $52.78 \%$ to $97.02 \%$ (Figure 3). As for WBMM, after applying the model, the percentage of residuals less than $2 \mathrm{~kg}$ increased from $38.29 \%$ to $85.91 \%$. The effect sizes and $95 \%$ confidence intervals (CIs) for the difference in WBMM were 2.49 (2.22-2.76) for men and 2.12 (1.91-2.33) for women. The respective values for ASMM were 2.26 (2.10-2.41) for men and 1.75 (1.65-1.87) for women.

We also calculated VIF, the reciprocal of the tolerance statistics, and found that none of the VIF values were $>10$, which supports our interpretation of the reliable estimation of the coefficients. Therefore, including BMI and fat percent estimated by BIA improved the BIC without hampering the diagnostic of residuals. The leverage plots for ASMM and WBMM are shown in Supplementary Figure S1.

[Whole body muscle mass]
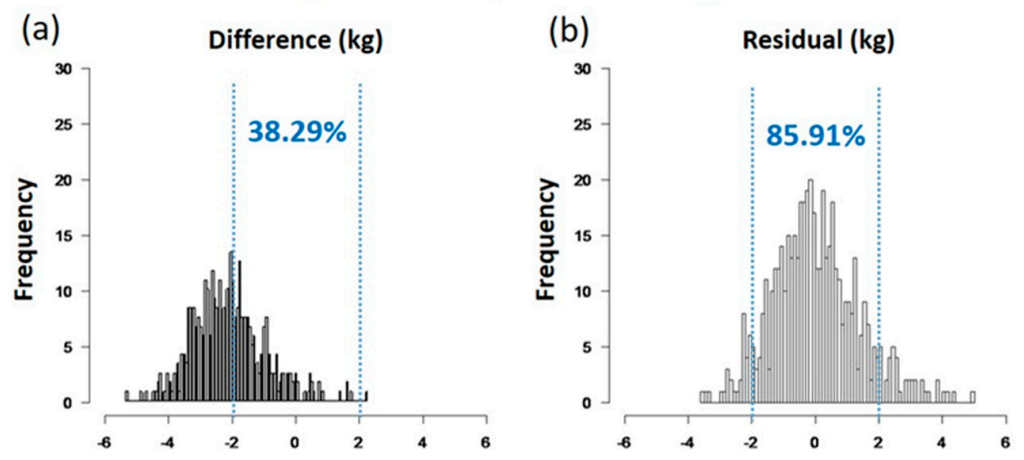

[Appendicular skeletal muscle mass]
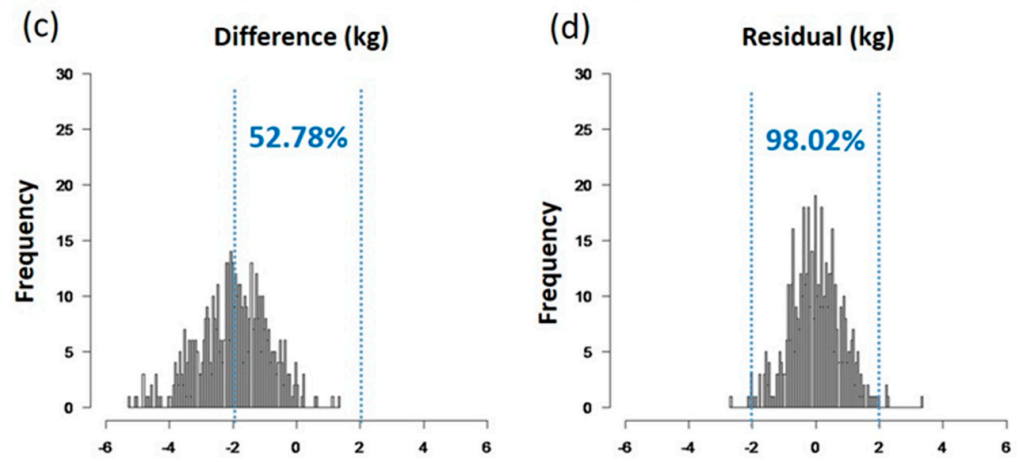

Figure 3. Comparing the percentages of residuals less than $2 \mathrm{~kg}$ between expectations before $(\mathbf{a}, \mathbf{c})$ and after $(\mathbf{b}, \mathbf{d})$ applying regression formulae. $(\mathbf{a}, \mathbf{c})$ WBMM; $(\mathbf{b}, \mathbf{d})$ ASMM. 
To validate the results from the regression models internally, we applied the bootstrapping method. The bootstrap standard errors of each variable were similar to those of the original models (Supplementary Table S2). The optimism average (bootstrap performance-test performance) values were also small (Supplementary Table S3). These data support the robustness of the original model. There was also no evidence of heteroscedasticity in the models when residual plots for ASMM and WBMM were checked visually (Supplementary Figures S2 and S3).

\section{Discussion}

In the present study, there was a high degree of correlation in muscle mass assessment between DXA and multifrequency-BIA methods in Korean adults both for WBMM and ASMM. The multifrequency-BIA method (Inbody770) overestimated the WBMM by $2.28 \mathrm{~kg}$ (95\% CI 2.11-2.45) and ASMM by $1.97 \mathrm{~kg}$ (95\% CI 1.87-2.06) compared with the DXA method (Hologic Horizon W).

Previous studies tried to validate BIA method in assessment of muscle mass with DXA. However, most studies were conducted with subjects of European origin [16,17]. In 1998, Pietrobelli et al. tried to validate a BIA estimate in the assessment of ASMM using height-adjusted appendicular impedance [31]. However, there were only 49 subjects, and inclusion of reactance failed to improve the estimation. In 2003, Kyle et al. tried to validate a prediction equation for estimating ASMM [16]. They suggested an equation based on reactance and resistance index (height ${ }^{2} / R$ ), and this improved the accuracy of ASMM estimation, particularly in older compared with younger subjects. However, they used a single frequency BIA machine. In 2015, Sergi et al. derived equations using resistance index and reactance for ASMM without considering age in their model [17]. Their equations showed an $r^{2}$ value of 0.92 in the estimation of ASMM. They suggested that the resistive index was the single best predictor of ASMM in older people. However, in that study, subjects with chronic comorbidities were excluded, which could limit the use of this equation in sarcopenic elderly persons [17].

There have been several studies on Asian ethnic groups [32,33]. In a study on Japanese, a new BIA equation from DXA-measured ASMM explained $87 \%$ of the BIA values in men and $89 \%$ in women using an equation including an impedance index [32]. However, only 250 subjects were studied and only elderly people over 65 years old were recruited. Another study with 720 Koreans analyzed a new model including the Height ${ }^{2}$ /resistance index for ASMM and found that it explained $82.5 \%$ of the variance [33]. However, they used an older BIA machine developed in 2002 and the study subjects were all elderly people aged over 65 years.

More recently, there have been several studies investigating sarcopenia with BIA methods $[26,34,35]$. A study on 756 Japanese individuals aged $18-86$ years attempted to generate the equations for ASMM using the traditional impedance index and an impedance ratio obtained from a BIA machine (MC-780A-N, TANITA, Tokyo, Japan) [34]. However, the correlation coefficients between ASMM values measured by DXA and those measured by BIA were unacceptable: 0.7 in men and 0.67 in women. Another study [35] showed that the accuracy of values from two advanced BIA machines differed: for the measurement of lean mass, there was $0.4 \mathrm{~kg}$ difference between DXA and an Inbody770 machine, but there was around a $5.5 \mathrm{~kg}$ difference between DXA and a TANIA980MA machine (TANITA). Another study on a Chinese population also found that the correlations between ASMM values obtained using the InBody720 (InBody, Seoul, Korea) BIA machine (which uses the same hardware as the InBody770) and those by DXA were high: the Pearson correlation coefficient and standard error of the estimate of the regression equation were 0.94 and $1.05 \mathrm{~kg}$ in men, and 0.90 and $0.93 \mathrm{~kg}$ in women, respectively [26].

In our study, with a wide age range, the actual differences between BIA and DXA estimates ranged from -5.55 to $8.10 \mathrm{~kg}$ for WBMM. We found that the difference in estimating WBMM between the two methods was affected by BMI, gender, and body fat. For example, subgroups with a lower BMI showed larger differences in muscle masses compared with their counterparts with a higher BMI. East Asians tend to have a lower muscle mass but higher fat percentage than Europeans for the same BMI [36]. The gap between BIA and DXA in Asian ethnic groups seems to be greater than 
that in Europeans or Africans [37]. These data suggest that ethnicity should be considered in the interpretation of BIA methods. From a different aspect, considering that WBMM includes trunk mass, which comprises much of the total muscle mass, more precise assessments in the estimations of muscle mass in the trunk or abdomen are needed.

There were greater differences in muscle mass assessment between the two methods in men than in women. This result suggests than muscle mass assessment by BIA is affected by gender and supports the need for gender-specific formulae for analyzing body composition. Body fluid status or DM might also affect muscle mass estimations through alterations in impedance [38,39]. In our study, anemia, measures of renal function by eGFR, presence of DM, and the use of diuretics or TZD did not alter the difference between two methods, which allowed us to use the BIA approach in this population.

\section{Strengths and Limitations}

The strength of this study is that the number of study subjects was larger than in previous studies [15-17,31,32]. We included a much wider range of adults, aged from 28 to 89 years, and investigated ASMM as well as WBMM. To use the validation equation practically, we included variables that were relevant and easily accessible, such as age, gender, BMI, and body fat from the BIA measurements. With this simplified formula, we improved the accuracy of BIA in muscle mass assessment and minimized the percentage of residuals within $2 \mathrm{~kg}$ (Figure 3). This improvement in accuracy might be able to help researchers estimate muscle mass more accurately using BIA methods. In addition, we analyzed subgroups according to physiological and disease status, such as anemia, kidney function, DM, and medications such as diuretics or TZD, which were not considered in previous studies. We derived specific equations for ASMM as well as WBMM, which are critical in the diagnosis of sarcopenia $[40,41]$.

However, there are several limitations to this study. First, this was not a community-based study and a non-probability convenience sampling method was used. Second, the participants were all Koreans. Therefore, the equations suggested in this study cannot be extrapolated to general populations or other ethnic groups and cross-validation is needed. However, we applied the bootstrapping method for the purpose of internal validation, and the result supports the robustness of the current study. Third, more variables are required to yield better accuracy of muscle mass estimation. To reduce the complexity of collecting additional information, we chose BMI, gender, and fat percentage data when building the regression equations because these can be easily obtained in clinical practice. Most companies selling BIA machines do not disclose the equations they use to estimate muscle mass, which makes it difficult to use them for research purposes [42]. We believe that incorporation of impedance values in regression equations is complicated and beyond the scope of this study. Fourth, because the BIA used in this study was developed for measuring body composition in the standing position, people who were bedridden, who had extremity amputation, or who could not hold the hand grip were not enrolled in this study. Lastly, the relative error was larger for ASMM measured by BIA than for WBMM measured by BIA, and this may have introduced a significant error in the assessment of sarcopenia based on appendicular skeletal muscle mass.

\section{Conclusions}

We found that muscle mass estimates by BIA were highly correlated with those measured by DXA. However, there was a tendency for the BIA method to overestimate muscle mass for DXA, particularly in men and in subjects with a low BMI. To increase accuracy in muscle mass assessment, we have developed regression equations with simple variables, which increased the percentage of residuals less than $2 \mathrm{~kg}$ to $>85 \%$ in WBMM and $>98 \%$ in ASMM. Although further validation is needed, we believe that these simplified and practical equations might offer an option to estimate muscle mass accurately at appendicular as well as in whole body levels. 
Supplementary Materials: The following are available online at http:/ / www.mdpi.com/2072-6643/10/6/738/s1, Figure S1: The leverage plots for ASMM and WBMM, Figure S2: A residual plot for ASMM, Figure S3: A residual plot for WBMM, Table S1: Gender-specific subgroup analyses of mean differences between DXA and BIA muscle mass estimates according to BMI and age categories, Table S2: Bias-corrected percentile intervals of residuals in bootstrapping method, Table S3: Optimism average (bootstrap performance-test performance) values.

Author Contributions: S.Y.L. and S.A. performed the primary analysis and drafted the manuscript. Y.J.K. and M.J.J. contributed to the data collection. K.M.K., S.H.C., and H.C.J. researched data and contributed to the discussion and review and editing of the manuscript. S.L. conceived the idea for the study, supervised the research and the statistical analyses, and reviewed and edited the manuscript. S.L. is the guarantor of this work and, as such, had full access to all the data in the study and takes responsibility for the integrity of the data and the accuracy of the data analysis.

Acknowledgments: This study was supported by a 2012 Moonsuk Research Grant from the Korean Society for the Study of Obesity, a grant of the Korean Society of CardioMetabolic Syndrome, and research funds from SNUBH.

Conflicts of Interest: The authors declare no conflicts of interest relevant to this manuscript.

\section{References}

1. Kim, J.H.; Lim, S.; Choi, S.H.; Kim, K.M.; Yoon, J.W.; Kim, K.W.; Lim, J.Y.; Park, K.S.; Jang, H.C. Sarcopenia: An independent predictor of mortality in community-dwelling older Korean men. J. Gerontol. A Biol. Sci. Med. Sci. 2014, 69, 1244-1252. [CrossRef] [PubMed]

2. Chen, L.K.; Liu, L.K.; Woo, J.; Assantachai, P.; Auyeung, T.W.; Bahyah, K.S.; Chou, M.Y.; Chen, L.Y.; Hsu, P.S.; Krairit, O.; et al. Sarcopenia in Asia: Consensus report of the Asian working group for sarcopenia. J. Am. Med. Dir. Assoc. 2014, 15, 95-101. [CrossRef] [PubMed]

3. Visser, M.; Schaap, L.A. Consequences of sarcopenia. Clin. Geriatr. Med. 2011, 27, 387-399. [CrossRef] [PubMed]

4. Baumgartner, R.N.; Koehler, K.M.; Gallagher, D.; Romero, L.; Heymsfield, S.B.; Ross, R.R.; Garry, P.J.; Lindeman, R.D. Epidemiology of sarcopenia among the elderly in new Mexico. Am. J. Epidemiol. 1998, 147, 755-763. [CrossRef] [PubMed]

5. Janssen, I. Influence of sarcopenia on the development of physical disability: The cardiovascular health study. J. Am. Geriatr. Soc. 2006, 54, 56-62. [CrossRef] [PubMed]

6. Janssen, I.; Heymsfield, S.B.; Ross, R. Low relative skeletal muscle mass (sarcopenia) in older persons is associated with functional impairment and physical disability. J. Am. Geriatr. Soc. 2002, 50, 889-896. [CrossRef] [PubMed]

7. Roubenoff, R. Sarcopenia and its implications for the elderly. Eur. J. Clin. Nutr. 2000, 54 (Suppl. 3), S40. [CrossRef] [PubMed]

8. Periasamy, M.; Herrera, J.L.; Reis, F.C.G. Skeletal muscle thermogenesis and its role in whole body energy metabolism. Diabetes Metab. J. 2017, 41, 327-336. [CrossRef] [PubMed]

9. Cawthon, P.M. Assessment of lean mass and physical performance in sarcopenia. J. Clin. Densitom. 2015, 18, 467-471. [CrossRef] [PubMed]

10. Thomas, D.R. Sarcopenia. Clin. Geriatr. Med. 2010, 26, 331-346. [CrossRef] [PubMed]

11. Buckinx, F.; Landi, F.; Cesari, M.; Fielding, R.A.; Visser, M.; Engelke, K.; Maggi, S.; Dennison, E.; Al-Daghri, N.M.; Allepaerts, S.; et al. Pitfalls in the measurement of muscle mass: A need for a reference standard. J. Cachexia Sarcopenia Muscle 2018. [CrossRef] [PubMed]

12. Bazzocchi, A.; Diano, D.; Ponti, F.; Andreone, A.; Sassi, C.; Albisinni, U.; Marchesini, G.; Battista, G. Health and ageing: A cross-sectional study of body composition. Clin. Nutr. 2013, 32, 569-578. [CrossRef] [PubMed]

13. Buckinx, F.; Reginster, J.Y.; Dardenne, N.; Croisiser, J.L.; Kaux, J.F.; Beaudart, C.; Slomian, J.; Bruyere, O. Concordance between muscle mass assessed by bioelectrical impedance analysis and by dual energy X-ray absorptiometry: A cross-sectional study. BMC Musculoskelet. Disord. 2015, 16, 60. [CrossRef] [PubMed]

14. Thibault, R.; Pichard, C. The evaluation of body composition: A useful tool for clinical practice. Ann. Nutr. Metab. 2012, 60, 6-16. [CrossRef] [PubMed]

15. Bosaeus, I.; Wilcox, G.; Rothenberg, E.; Strauss, B.J. Skeletal muscle mass in hospitalized elderly patients: Comparison of measurements by single-frequency bia and dxa. Clin. Nutr. 2014, 33, 426-431. [CrossRef] [PubMed] 
16. Kyle, U.G.; Genton, L.; Hans, D.; Pichard, C. Validation of a bioelectrical impedance analysis equation to predict appendicular skeletal muscle mass (asmm). Clin. Nutr. 2003, 22, 537-543. [CrossRef]

17. Sergi, G.; De Rui, M.; Veronese, N.; Bolzetta, F.; Berton, L.; Carraro, S.; Bano, G.; Coin, A.; Manzato, E.; Perissinotto, E. Assessing appendicular skeletal muscle mass with bioelectrical impedance analysis in free-living caucasian older adults. Clin. Nutr. 2015, 34, 667-673. [CrossRef] [PubMed]

18. Bohm, A.; Heitmann, B.L. The use of bioelectrical impedance analysis for body composition in epidemiological studies. Eur. J. Clin. Nutr. 2013, 67 (Suppl. 1), S79. [CrossRef] [PubMed]

19. Kyle, U.G.; Bosaeus, I.; De Lorenzo, A.D.; Deurenberg, P.; Elia, M.; Gomez, J.M.; Heitmann, B.L.; Kent-Smith, L.; Melchior, J.C.; Pirlich, M.; et al. Bioelectrical impedance analysis—Part I: Review of principles and methods. Clin. Nutr. 2004, 23, 1226-1243. [CrossRef] [PubMed]

20. Janssen, I.; Heymsfield, S.B.; Baumgartner, R.N.; Ross, R. Estimation of skeletal muscle mass by bioelectrical impedance analysis. J. Appl. Physiol. 2000, 89, 465-471. [CrossRef] [PubMed]

21. Rangel Peniche, D.B.; Raya Giorguli, G.; Aleman-Mateo, H. Accuracy of a predictive bioelectrical impedance analysis equation for estimating appendicular skeletal muscle mass in a non-caucasian sample of older people. Arch. Gerontol. Geriatr. 2015, 61, 39-43. [CrossRef] [PubMed]

22. Scott, D.; Park, M.S.; Kim, T.N.; Ryu, J.Y.; Hong, H.C.; Yoo, H.J.; Baik, S.H.; Jones, G.; Choi, K.M. Associations of low muscle mass and the metabolic syndrome in caucasian and asian middle-aged and older adults. J. Nutr. Health Aging 2016, 20, 248-255. [CrossRef] [PubMed]

23. Haldar, S.; Chia, S.C.; Henry, C.J. Body composition in asians and caucasians: Comparative analyses and influences on cardiometabolic outcomes. Adv. Food Nutr. Res. 2015, 75, 97-154. [PubMed]

24. Mente, A.; Razak, F.; Blankenberg, S.; Vuksan, V.; Davis, A.D.; Miller, R.; Teo, K.; Gerstein, H.; Sharma, A.M.; Yusuf, S.; et al. Ethnic variation in adiponectin and leptin levels and their association with adiposity and insulin resistance. Diabetes Care 2010, 33, 1629-1634. [CrossRef] [PubMed]

25. Glickman, S.G.; Marn, C.S.; Supiano, M.A.; Dengel, D.R. Validity and reliability of dual-energy X-ray absorptiometry for the assessment of abdominal adiposity. J. Appl. Physiol. 2004, 97, 509-514. [CrossRef] [PubMed]

26. Wang, H.; Hai, S.; Cao, L.; Zhou, J.; Liu, P.; Dong, B.R. Estimation of prevalence of sarcopenia by using a new bioelectrical impedance analysis in chinese community-dwelling elderly people. BMC Geriatr. 2016, 16, 216. [CrossRef] [PubMed]

27. Gibson, A.L.; Holmes, J.C.; Desautels, R.L.; Edmonds, L.B.; Nuudi, L. Ability of new octapolar bioimpedance spectroscopy analyzers to predict 4-component-model percentage body fat in hispanic, black, and white adults. Am. J. Clin. Nutr. 2008, 87, 332-338. [CrossRef] [PubMed]

28. Lee, D.H.; Park, K.S.; Ahn, S.; Ku, E.J.; Jung, K.Y.; Kim, Y.J.; Kim, K.M.; Moon, J.H.; Choi, S.H.; Park, K.S.; et al. Comparison of abdominal visceral adipose tissue area measured by computed tomography with that estimated by bioelectrical impedance analysis method in korean subjects. Nutrients 2015, 7, 10513-10524. [CrossRef] [PubMed]

29. Park, K.S.; Lee, D.H.; Lee, J.; Kim, Y.J.; Jung, K.Y.; Kim, K.M.; Kwak, S.H.; Choi, S.H.; Park, K.S.; Jang, H.C.; et al. Comparison between two methods of bioelectrical impedance analyses for accuracy in measuring abdominal visceral fat area. J. Diabetes Complicat. 2016, 30, 343-349. [CrossRef] [PubMed]

30. Lukaski, H.C.; Bolonchuk, W.W.; Hall, C.B.; Siders, W.A. Validation of tetrapolar bioelectrical impedance method to assess human body composition. J. Appl. Physiol. 1986, 60, 1327-1332. [CrossRef] [PubMed]

31. Pietrobelli, A.; Morini, P.; Battistini, N.; Chiumello, G.; Nunez, C.; Heymsfield, S.B. Appendicular skeletal muscle mass: Prediction from multiple frequency segmental bioimpedance analysis. Eur. J. Clin. Nutr. 1998, 52,507-511. [CrossRef] [PubMed]

32. Yoshida, D.; Shimada, H.; Park, H.; Anan, Y.; Ito, T.; Harada, A.; Suzuki, T. Development of an equation for estimating appendicular skeletal muscle mass in japanese older adults using bioelectrical impedance analysis. Geriatr. Gerontol. Int. 2014, 14, 851-857. [CrossRef] [PubMed]

33. Kim, J.H.; Choi, S.H.; Lim, S.; Kim, K.W.; Lim, J.Y.; Cho, N.H.; Park, K.S.; Jang, H.C. Assessment of appendicular skeletal muscle mass by bioimpedance in older community-dwelling korean adults. Arch. Gerontol. Geriatr. 2014, 58, 303-307. [CrossRef] [PubMed] 
34. Yamada, Y.; Nishizawa, M.; Uchiyama, T.; Kasahara, Y.; Shindo, M.; Miyachi, M.; Tanaka, S. Developing and validating an age-independent equation using multi-frequency bioelectrical impedance analysis for estimation of appendicular skeletal muscle mass and establishing a cutoff for sarcopenia. Int. J. Environ. Res. Public Health 2017, 14, 809. [CrossRef] [PubMed]

35. Alkahtani, S.A. A cross-sectional study on sarcopenia using different methods: Reference values for healthy Saudi young men. BMC Musculoskelet. Disord. 2017, 18, 119. [CrossRef] [PubMed]

36. Kumar, S.; Khosravi, M.; Massart, A.; Potluri, M.; Davenport, A. The effects of racial differences on body composition and total body water measured by multifrequency bioelectrical impedance analysis influence delivered kt/v dialysis dosing. Nephron Clin. Pract. 2013, 124, 60-66. [CrossRef] [PubMed]

37. Bosy-Westphal, A.; Jensen, B.; Braun, W.; Pourhassan, M.; Gallagher, D.; Muller, M.J. Quantification of whole-body and segmental skeletal muscle mass using phase-sensitive 8-electrode medical bioelectrical impedance devices. Eur. J. Clin. Nutr. 2017, 71, 1061-1067. [CrossRef] [PubMed]

38. Yanovski, S.Z.; Hubbard, V.S.; Heymsfield, S.B.; Lukaski, H.C. Bioelectrical impedance analysis in body composition measurement: National institutes of health technology assessment conference statement. Am. J. Clin. Nutr. 1996, 64, S524-S532.

39. Jang, H.C. Sarcopenia, frailty, and diabetes in older adults. Diabetes Metab. J. 2016, 40, 182-189. [CrossRef] [PubMed]

40. Studenski, S.A.; Peters, K.W.; Alley, D.E.; Cawthon, P.M.; McLean, R.R.; Harris, T.B.; Ferrucci, L.; Guralnik, J.M.; Fragala, M.S.; Kenny, A.M.; et al. The fnih sarcopenia project: Rationale, study description, conference recommendations, and final estimates. J. Gerontol. A Biol. Sci. Med. Sci. 2014, 69, 547-558. [CrossRef] [PubMed]

41. Kim, K.M.; Jang, H.C.; Lim, S. Differences among skeletal muscle mass indices derived from height-, weight-, and body mass index-adjusted models in assessing sarcopenia. Korean J. Intern. Med. 2016, 31, 643-650. [CrossRef] [PubMed]

42. Gonzalez, M.C.; Heymsfield, S.B. Bioelectrical impedance analysis for diagnosing sarcopenia and cachexia: What are we really estimating? J. Cachexia Sarcopenia Muscle 2017, 8, 187-189. [CrossRef] [PubMed]

(C) 2018 by the authors. Licensee MDPI, Basel, Switzerland. This article is an open access article distributed under the terms and conditions of the Creative Commons Attribution (CC BY) license (http://creativecommons.org/licenses/by/4.0/). 